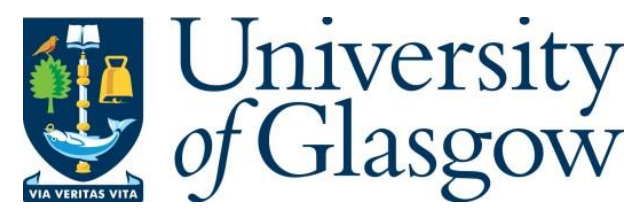

Yuan, M., Ghannam, R., Karadimas, P. and Heidari, H. (2019) Flexible RFID Patch for Food Spoilage Monitoring. In: IEEE Asia Pacific Conference on Postgraduate Research in Microelectronics and Electronics (PrimeAsia 2018), Chengdu, China, 26-30 Oct 2018, pp. 68-71. ISBN 9781538695913.

There may be differences between this version and the published version. You are advised to consult the publisher's version if you wish to cite from it.

http://eprints.gla.ac.uk/168942/

Deposited on: 14 September 2018

Enlighten - Research publications by members of the University of Glasgow http://eprints.gla.ac.uk 


\title{
Flexible RFID Patch for Food Spoilage Monitoring
}

\author{
Mengyao Yuan, Rami Ghannam, Petros Karadimas and Hadi Heidari \\ School of Engineering, University of Glasgow, G12 8QQ, UK \\ hadi.heidari@glasgow.ac.uk
}

\begin{abstract}
When food is reversed under an inappropriate situation, for example inadequate temperature, foodstuff would turn rotten as a result of the rapid breed of food spoilage bacteria under warm and wet circumstances where bacteria can be easily generated, the consequences can be worse if human beings eat those degenerative comestible, we might get bromatoxism. This paper focuses on the designing and simulation of a wearable radio frequency identification (RFID) patch for food spoilage monitoring with smart packaging that can be recognized and read temperature information by device supporting near field communication (NFC) technology through an attached circular antenna. More precisely, this patch can be read under a designed high frequency of $13.56 \mathrm{MHz}$, and data is transmitted from the chip MLX90129 with its internal sensor that sends the temperature to the reader module.
\end{abstract}

\section{Keywords- Temperature Sensing; RFID, Smart Packaging; Food Monitoring.}

\section{INTRODUCTION}

Shelf life is the definition of longest recommended time for product or fresh production that can be stored, and in the meantime, illustrates the expected conditions during distribution and storage in order to maintain the product quality. The expiration date usually depends on the degradation mechanism of the specific product, which may be affected by a variety of factors: exposure to light, heat, moisture, gas transmission, mechanical stress, and contamination with substances such as microorganisms. Thus, the measurement for safety food becomes the key concern, which is also the main achievement of this project by censoring the temperature.

RFID has been widely used in automatic identification rather than barcode technology, which was used to be the most generalized automatic identification developed in the 1970s[1]. It is a technology that uses specific recognition devices to automatically obtain the related information of the identified objects through the close action between them, which at the same time sends the information it carries to a computer processing system working on the backstage for further analysis. A RFID patch is a tag supporting wireless communicating technology that can identify specific targets and read and write data through radio signals without identifying mechanical or optical contact between the system and a particular target [2]. In the course of the process, the radio signal is transmitted from the label, attached to the object, through the radio frequency electromagnetic field for automatically

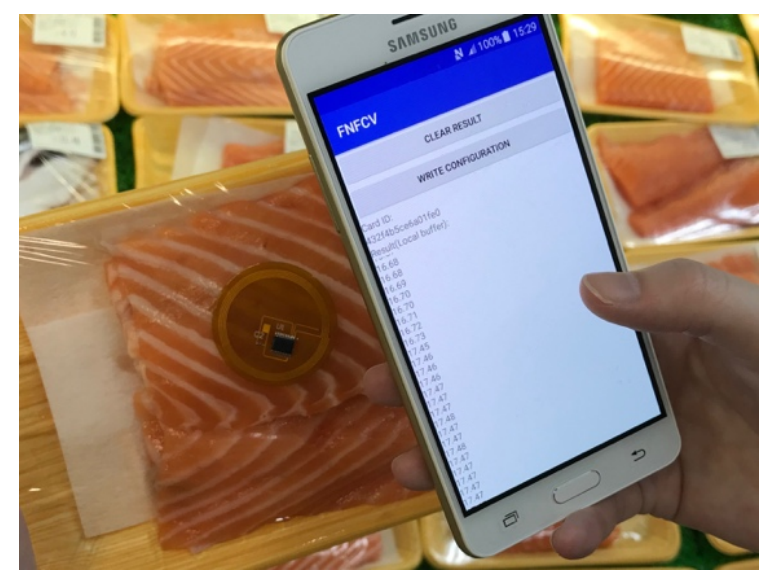

Fig. 1. Reading Salmon temperature through RFID.

identifying and tracking the item. RFID patch is used in quite a few industries currently. In terms of this project, the patch is used to monitor the food temperature which illustrates the spoilage stage. The basic characteristics for the patch are the wearability, waterproofness, and flexibility.

\section{TeChNOLOGY SuPPORT}

In general, RFID is categorized by the transmitting frequency varying from $100 \mathrm{KHz}$ to $2.45 \mathrm{GHz}$, which is distributed as Low Frequency, High Frequency, Ultra High Frequency and Microwave, as is shown in Table 1. Taking their identification speed, environmental reaction and tag size into consideration, high frequency is chosen for its adequate reading distance and attainable small size. As for radio frequency of $13.56 \mathrm{MHz}$, Near Field Communication (NFC) Technology also known as proximity wireless communication is the main technology which allows non-contact point-to-point data transmission between electronic devices to exchange data[3]. The compatibility of this technology is divided into two main categories, ISO 14443 and ISO 15693, the former one realizes higher data speed up to $106 \mathrm{Kbit} / \mathrm{s}$ but the reading distance is restricted within $10 \mathrm{~cm}$, which is called proximity card while the standard ISO 15693 that been used in this project can magnify the distance to $10 \mathrm{~cm}-1 \mathrm{~m}$ and it is called vicinity card.

Besides the communication technology, chip MLX90129 produced by Melexis Company which combines a precise acquisition chain for external resistive sensors with a wide range of interface possibilities is used as the RFID and temperature sensor. Without any other components than a $13.56 \mathrm{MHz}$ tuned antenna, it becomes a RFID temperature sensor. For measuring other physical parameters, one or two resistive sensors can be connected to make battery-less sensing 
point. The sensor output data is stored in the internal 3.5kbits user memory. The measurement range for this chip is from $40^{\circ} \mathrm{C}$ to $105^{\circ} \mathrm{C}$, and the circuit design for the MLX90129 chip when it operates as a temperature sensor during RFID is shown below, where pin 1 and pin 2 are connected to the antenna and a capacitor with $100 \mathrm{nF}$ capacitance should be connected with pin3, the VFIELD pin, and the VSS pin19 needs to be connected with ground [4].

Table 1. RFID Classification

\begin{tabular}{|c|c|c|c|c|c|}
\hline \multirow{2}{*}{ Frequency } & $\begin{array}{c}\text { Low } \\
\text { Frequency } \\
\text { (LF) }\end{array}$ & $\begin{array}{c}\text { High } \\
\text { Frequency } \\
\text { (HF) }\end{array}$ & \multicolumn{2}{|c|}{ Ultra High Frequency(UHF) } & Microwave \\
\cline { 2 - 6 } & $125.124 \mathrm{KHz}$ & $13.56 \mathrm{MHz}$ & $433.92 \mathrm{MHz}$ & $860-960 \mathrm{MHz}$ & $2.45 \mathrm{GHz}$ \\
\hline $\begin{array}{c}\text { Identification } \\
\text { distance }\end{array}$ & $10 \mathrm{~cm}$ & $10 \mathrm{~cm}-1 \mathrm{~m}$ & $50-100 \mathrm{~m}$ & $\begin{array}{c}3.5-5 \mathrm{~m}(\mathrm{P}) \\
1-100 \mathrm{~m}(\mathrm{~A})\end{array}$ & $\begin{array}{c}1-2 \mathrm{~m}(\mathrm{P}) \\
1-50 \mathrm{~m}(\mathrm{~A})\end{array}$ \\
\hline $\begin{array}{c}\text { Operation } \\
\text { type }\end{array}$ & Passive & Passive & Active & Passive/Active & Passive/Active \\
\hline $\begin{array}{c}\text { Identification } \\
\text { speed }\end{array}$ & Low & & & high \\
\hline $\begin{array}{c}\text { Environmental } \\
\text { reaction }\end{array}$ & Slow $\longrightarrow$ rapid \\
\hline Tag size & Small & & & \\
\hline
\end{tabular}

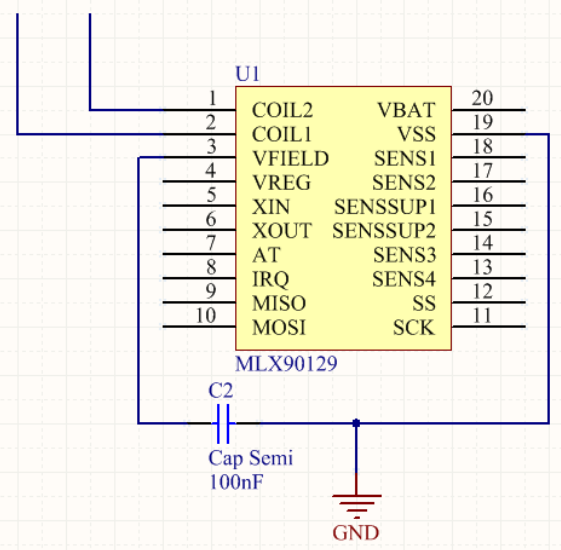

Fig. 2. Circuit design with chip MLX90129.

\section{Design And Implementation Process}

An antenna is a device that receives or radiates radio frequency signals of radio transceivers in the form of electromagnetic waves, where the interface between free space and transmission line is an indispensable part of wireless communication system[5]. For NFC antenna design, the relation of resonance frequency, inductance and capacitance is defined as follows

$$
f_{0}=\frac{1}{2 \pi \sqrt{L C}}
$$

In this case, resonance frequency should be $13.56 \mathrm{MHz}$, and the inner capacitance of the MLX90129 chip is $75 \mathrm{pF}$, thus the inductance of the antenna should be designed as $1.837 \mu \mathrm{H}$.

For spiral shape antenna or circular antenna, the computing formula is defined as

$$
L_{\text {coil }}=\frac{0.3937 \cdot(a \cdot N)^{2}}{8 \cdot a+11 \cdot b}
$$

In this case, $\mathrm{a}=\left(r_{i}+r_{o}\right) / 2$ and $\mathrm{b}=r_{o}-r_{i}, r_{i}$ is the radius of the inner circular antenna, while $r_{o}$ is the outer radius, and $N$ is denoted as the number of turns. During this project, HFSS is used as the simulation software of the antenna design, in order to apply a spiral shape, the Archimedes Spiral Antenna is chosen to be the basic shape. The radius of the Archimedes spiral antenna increases evenly with the Angle, which can be defined as:

$$
\mathrm{r}=r_{0}+a \phi
$$

Where $r_{0}$ is the starting radius, a is the growth rate of spiral, and $\phi$ denotes the angle or the arc.

To a large extent, the performance of NFC communication depends on the performance of tags, where tag antenna is one of the dominating factors that determine the performance of them[6]. The input impedance of the antenna is measured by network analyser to describe the input characteristic of the antenna for the most part[7]. As the terminal load of the transmission line, the antenna can be regarded as a twoterminal network, and the input impedance of the antenna is defined as the ratio of the input voltage to the current, denoted as

$$
Z_{A}=\frac{U_{\text {in }}}{I_{\text {in }}}=R_{A}+j X_{A}
$$

In accordance with the principle of Archimedes Spiral antenna design, the relation of the radius and the angle increment could be transformed into Cartesian coordinates, and can be written with functions in each axis, as a result, functions are defined as

$$
\begin{aligned}
& x_{(t)}=r_{0} e^{a t} \cos (t) \\
& y_{(t)}=r_{0} e^{a t} \sin (t)
\end{aligned}
$$

The start point is set to 0 which means that the loop starts from zero point in the axis, and the end point is defined with parameter fi which is set to 30 , representing the stop angle of the antenna.

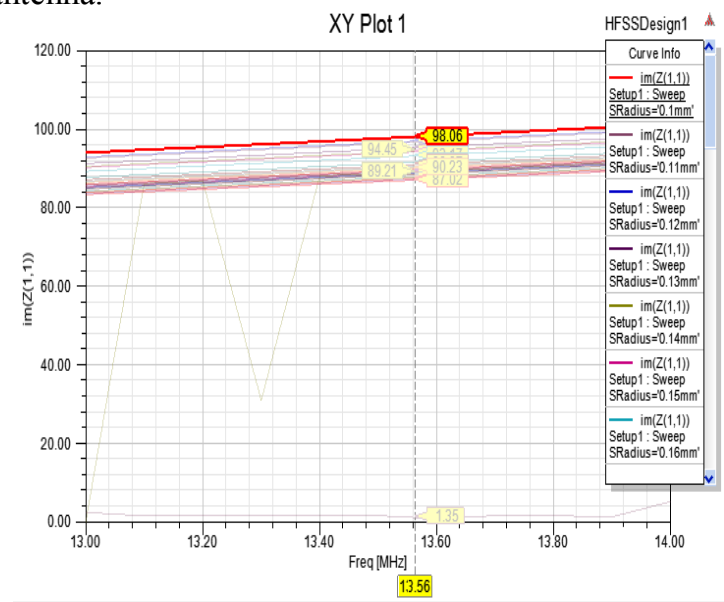

Figure 3. Simulation of the leading factor

As we can see from the simulation of the parameters, it is shown that with a fixed inner radius and outer radius, the cardinal influence of the inductance of the loop goes to the width of the wire, normally, the width should be in the range of $0.2 \mathrm{~mm}$ to $0.5 \mathrm{~mm}$ for NFC antenna, from the swept parameter result, $0.2 \mathrm{~mm}$ meet the required inductance closely. By the applying functions and parameters, the antenna size is designed with $15 \mathrm{~mm}$ inner radius considering the size of the 
chip MLX90129, 17.5mm outer radius for phone recognition with 5 coil winding to increase the inductance of the antenna.

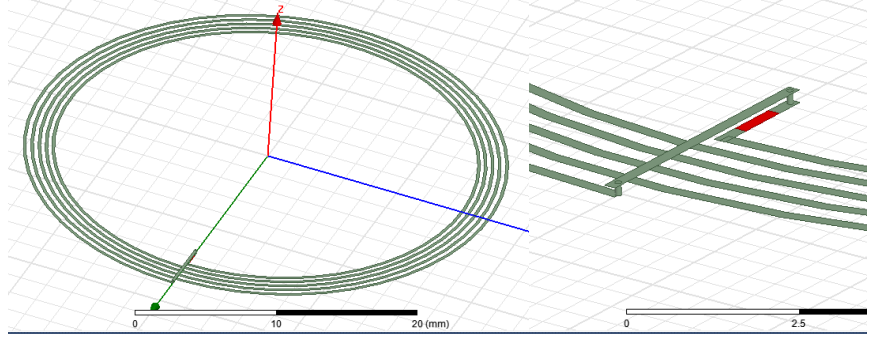

Figure 4. (a) Spiral antenna, (b) lump port as the chip

As is shown above, the antenna is designed to a circular form using functions that are mentioned before, and the right side diagram illustrates the connection of the inner radius and the outer one with two cylindrical box and the red rectangular is the replacement of chip in the simulation software, which aims to insert current flows to the antenna.
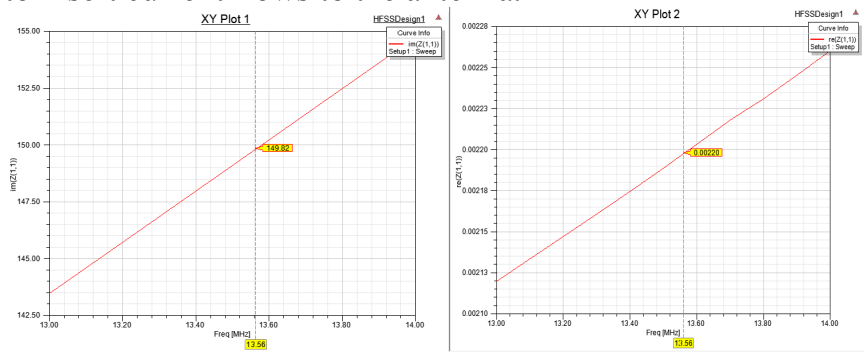

Figure 5. Simulation results relating to (a) Inductance (b) Resistance

From Figure4, the imaginary part of parameter $\mathrm{Z}$ is shown and it refers to the calculation of the inductance by the formula:

$$
\mathrm{L}=\frac{\operatorname{im}(Z)}{2 \pi f_{0}}
$$

During which the inductance for the designed antenna is $1.757 \mu \mathrm{H}$ with a relative error of $4.35 \%$. Nevertheless, the real part of $\mathrm{Z}$ parameter for the designed the antenna is $0.00220 \Omega$ which turns to be a perfect value for resistance for NFC antenna. Nevertheless, in the NFC antenna design, the matching circuit is not necessary for the reason that the inductance of the antenna is designed for the signals transmitting under the same frequency and to maximize the efficiency.

The software Altium is used for the PCB layout design, the layout for the circuit can be generated directly from the schematic, and the antenna design could be imported from software HFSS for the antenna is constructed by function not with measurable radius and angles.

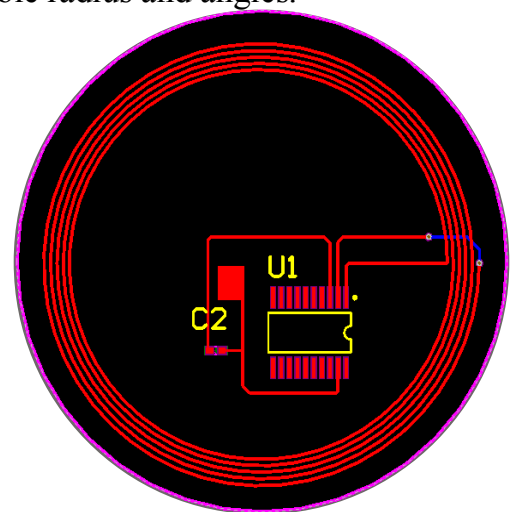

Figure 6. Printed Circuit Board (PCB) Layout.
There are few applications that can be used for tag identification in the current App market for NFC recognition, and the most typical two Apps are called 'NFCTools' and 'NFC Taginfo', both Apps can identify the type and the UID of the tag with the RF technology sometimes, but without the DSFID, where the data are stored in the blocks that should be read. After multiple time failures of finding the proper App for this chip, even with the contact with the chip supplier, I decided to make an applicable one instead of finding one. The main code for initialization is shown as below:

Table 2. Main Android set up code

\begin{tabular}{|l|c|c|}
\hline Address & hex & bit \\
\hline $0 \times 12$ & $00 \mathrm{FF}$ & 0000000011111111 \\
\hline $0 \times 15$ & 3773 & 0011011101110011 \\
\hline $0 \times 16$ & 0000 & 0000000000000000 \\
\hline $0 \times 17$ & 0000 & 0000000000000000 \\
\hline $0 \times 18$ & 0000 & 0000000000000000 \\
\hline $0 \times 19$ & 0231 & 0000001000110001 \\
\hline $0 \times 1 \mathrm{~A}$ & 8000 & 1000000000000000 \\
\hline
\end{tabular}

From the datasheet of MLX90129 we can conclude that $\# 12$ is the sensor's power configuration words, \#15 is for the sensor control word, \#16 is for the sensor 0, in other words, temperature sensor inside low threshold which need to be set to $0 x 00$. Similarly, the \#17 is also for the low threshold word and should be set to $0 \times 00$ as well, \#19 is for the sensor connection word and \#1A is for the sensor serial resistance configuration word.

\section{EXPERIMENTAL RESULTS AND DISCUSSION}

The pictures of the patch containing the chip and antenna with Polyethylene Terephthalate (PET) material and Polyimide are shown as below, it is clear that the circular antenna and the transparent or translucent materials increase the appreciability.

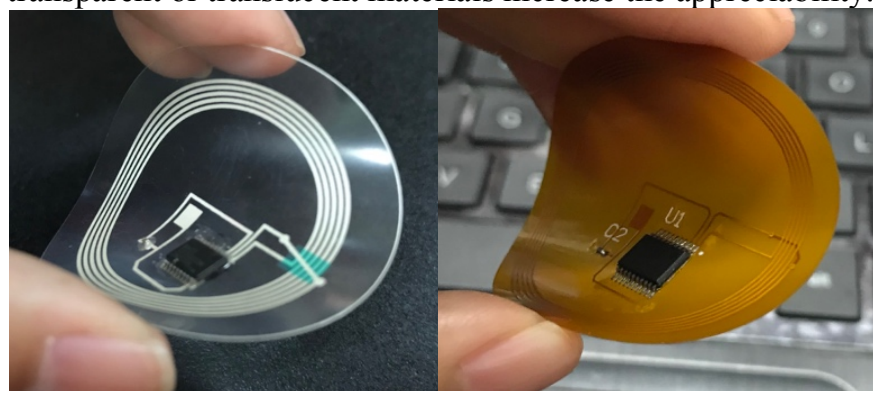

Figure 7. RFID patch (a) Transparent PET (b) translucent FPC

It turns out that for PET material, although the outlook is pretty, the performance of it is not so good. First of all, it can be recognized by Android Apps like NFC Taginfo or NFC Tools with the information of UID, Tag supporting protocol, $\mathrm{RF}$ technology which is also known as the patch type, and its serial number can also be displayed on the screen. The only problem is with the DSFID display that it is recognized as 0x00 
from both Apps compared with the information of our student ID. But for the self-designed software FNFCV, the temperature of the meat is measured by the translucent tag and displayed on the screen. Besides, the reading time for both tags is about 4 seconds, and for the transparent PET, it should be put near the antenna of the reader accurately while the translucent FPC tag should be put only within $3 \mathrm{~cm}$ or less.

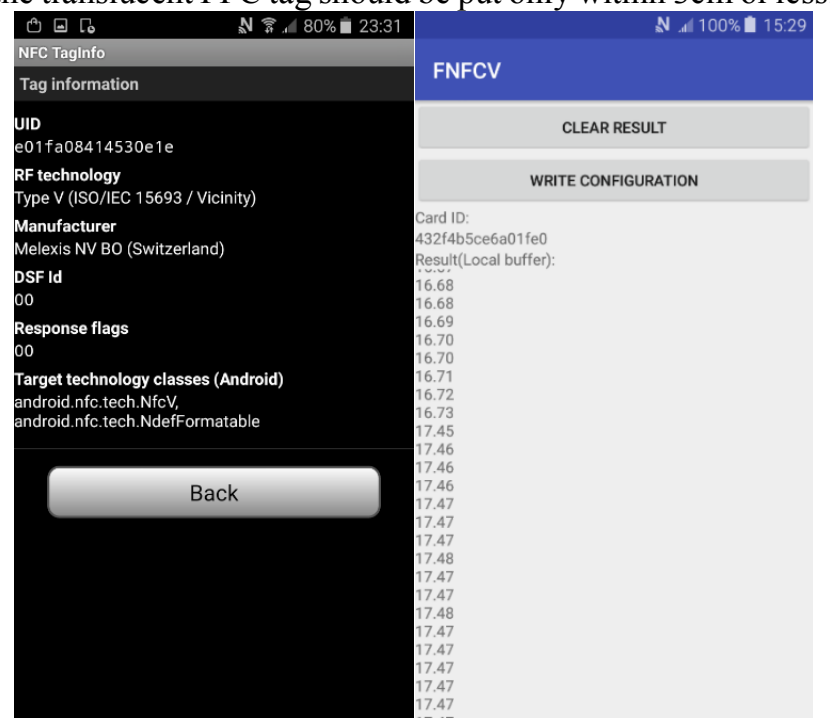

Figure 8. Reading result from (a) NFC Taginfo (b) FNFCV After the Smith diagram Analysis, the inductance of the transparent antenna is about $1.5117 \mu \mathrm{H}$, however the resistance of the antenna reaches $162.2 \Omega$, which should be about $0 \Omega$ for NFC design. On the contrary, the inductance of the translucent FPC is $1.985 \mu \mathrm{H}$, and the resistance of the antenna is $3.9 \Omega$, which is way smaller than the transparent one and results in less loss. However, this diversity resistance places a vital important role in the performance of the tag. The conductive material of the antenna is made of silver with conductive glued, silver material is similar with copper which is normally applied for flexible printed circuit board but with lower electrical resistivity and higher electrical conductivity. The resistance of it is tested within equal increment, and it turns out that the resistance of the antenna is increased with the tested length increase, which leads to the loss of the Quality factor of the antenna [8]. However, the bad antenna working might due to the temperature when transmitting, indeed, antenna would be affected through the change of temperature. The main reason for the influence of temperature on the propagation path is the boundary condition of the temperature layer, but only applied for the frequency with shorter wavelength.

\section{CONCLUSION AND OUTLOOK}

The project goal is to design a wearable RFID patch with a circular antenna and smart packaging. In order to achieve those goals, the RFID chip MLX90129 is chosen with the inner temperature sensor inside, software such as HFSS and Altium are used for the simulation of antenna design and the PCB drawing, moreover, Android Apps such as NFC Tools and NFC Taginfo are used to identify the patch and an App named FNFCV is designed to display the temperature result.
Generally, the spiral antenna and flexible printed circuit board with transparent PET and translucent outlook have been fabricated successfully, and the result shows that the translucent one could be recognized by Apps on phone and display the temperature successfully with limited reading time and distance.

The analysed cause for this problem goes to materials that contribute to the patch, for manufacturing method and actual conducting material varies a lot for different material. To name only a few, in order to demonstrate a transparent outlook, the substrate that is usually used is PVC or else PET which cannot survive under high temperature, thus this kind of material increases the complexity of welding or sticking technology. Secondly, considering the cost of printing silver material, companies usually use silver with glued as the conductive material, which in this case hugely affected the performance of the RFID patch.

In terms of the further improvement, there are two main aspects that may contribute to the integrity. First of all, the shape and size of the antenna can be altered to make the outlook more beautiful, such as round rectangular one with smaller sizes[9]. However, if the shape or the size has been changed, the design should be treated really carefully, for the inductance of the antenna may vary with the size in a great range. And the layout of the chip with the antenna should be placed properly to decrease the influence from the circuit. Moreover, the circuit could be designed to realize more functions such as testing humidity, gas intensity, or illumination intensity with different sensors attached to the chip.

\section{REFERENCES}

[1] Abanob Abdelnour, et al., "Transformation of Barcode Into RFID Tag, Design, and Validation" IEEE Microwave and Wireless Components Letters, vol. 28, pp. 398 - 400, May 2018.

[2] K. O. Htet, et al., "Switched Capacitor DC-DC Converter for Miniaturised Wearable Systems," in 2018 IEEE International Symposium on Circuits and Systems (ISCAS), 2018, pp. 1-5.

[3] V. Nabaei, et al., "Magnetic Biosensors: Modelling and Simulation," Biosensors and Bioelectronics, vol. 103, pp. 69-86, 2018.

[4] Melexis (n.d). Datasheet for MLX90129 [Online], Available: https://www.melexis.com/en/documents/documentation/datasheets/ mlx90129

[5] Albert Sabban, "Small wearable antennas for wireless communication and medical systems" Radio and Wireless Symposium (RWS), ISSN 2164-2974, March 2018.

[6] S. Wen, H. Heidari, et al., "A Wearable Fabric-Based RFID Skin Temperature Monitoring Patch," Proc. of IEEE Sensors Conference, 2016.

[7] A. Vilouras, et al., "Modeling of CMOS Devices and Circuits on Flexible Ultrathin Chips," IEEE Transactions on Electron Devices, vol. 64, pp. 2038-2046, 2017.

[8] Miloslav Capek, et al., "Minimization of Antenna Quality Factor" IEEE Transactions on Antennas and Propagation, vol. 65, pp. 4115 - 4123, June 2017.

[9] H. Heidari, et al., "Towards bendable CMOS magnetic sensors," in IEEE Conf. PhD Research Microelect. Elect. (PRIME), 2015, pp. 314-317. 